\title{
Constitutive Modelling of Geomaterials
}

\author{
Tejas G. Murthy ${ }^{1} \cdot$ Arghya Das $^{2}$
}

Published online: 2 August 2019

(C) Indian Geotechnical Society 2019

Establishment of an explicit relationship between stress and strain (load and deformation) has been the holy grail of modelling mechanical behaviour of materials. Geomaterials have very complex and interesting mechanical behaviour including pressure dependence, time dependence, fracture, and severe plastic deformation accompanied by volume change, very small range of elastic behaviour. In the past, an extreme reliance of empiricism was the norm, and modelling mechanical behaviour of geomaterials with a strong theoretical underpinning has been possible, especially after the 1960s. Folding in the basic tenet of the mathematical theory of plasticity into a framework of critical state soil mechanics revolutionized constitutive modelling of geomaterials. The implementation of such complex continuum models into design has also made engineering of foundations and earth structures optimal and a lot more reliable.

The ambit of what can be considered a geomaterial (such as landfills, composites including geotextiles-soils, reinforced earth) is ever increasing. In the recent times, the introduction of hypoplastic and hyperplastic frameworks has enabled tackling materials of complex constitutivity for applications beyond just infrastructure to areas such as energy storage, waste management, mining, and powder technology. Additionally, migration towards the discrete

Tejas G. Murthy

tejas@iisc.ac.in

Arghya Das

arghya@iitk.ac.in

1 Indian Institute of Science, Bangalore, India

2 Indian Institute of Technology, Kanpur, India element, material point, and particle hydrodynamics approaches for a multiple-length-scale understanding of the mechanical behaviour have not only increased the challenges in modelling, but also provided a deeper understanding of material behaviour. Important advances continue to be made in the geomechanics, soft condensed matter physics, rheology, and geoengineering communities on modelling of geomaterials. With the objective of highlighting recent trends in constitutive modelling and implementation, the Indian Geotechnical Journal is bringing forth this special issue on "Constitutive Modelling of Geomaterials".

This issue comprises seven articles that encompass constitutive modelling of geomaterials highlighting various aspects such as stress and material anisotropy, multiphysical effects, micromechanics, and numerical implementation.

It is well known that stress-induced anisotropy affects the fabric of clay. Chen et al. propose a rotational hardening model that reorients the yield surface as a function of loading history accounting for the effects of stress-induced anisotropy in clay. Fuentes and Lascarro address the importance of experimental methodology and consequent fabric evolution on the response of geomaterials. Such an effect is cast within a hypoplastic framework that captures the material anisotropy induced during cylindrical sample preparation for triaxial experiments. Multi-physical effects such as chemical, thermal, and hydraulic play a crucial role in the deformation response of various geoengineering problems (nuclear waste management, geothermal energy, and chemical weathering).

Shirasb et al. incorporate such effects within the critical state framework. In this study, thermal creep on sandbentonite mixture is modelled. 
Numerical analysis forms an inexorable part of constitutive modelling and tackling boundary value problems. Singh et al. focus on the numerical aspect of implementing constitutive model for solving boundary value problems. The analysis indicates that the coupling between solid and fluid phases of geomaterial holds a key for achieving a converged solution in commercial finite element codes, especially during localized deformation. Huang et al. demonstrate a numerical scheme that considers the role of constitutive modelling at two different length scales. A coupled continuum and discrete model is proposed for analysing the deformation in rock around an underground opening. Singh et al., on the other hand, proposed a closedform analytic solution of stress around circular opening using Drucker-Prager failure criteria.

While plasticity theory and its various manifestations have dominated the landscape of constitutive modelling in geomaterials, elasticity has always played a crucial role in efficiently model the response of geomaterials. $\mathrm{Hu}$ et al. use poroelasticity that was devised from Biot's formulation to simulate the dynamic response of saturated geomaterials.

While these papers offer a very small insight into the burgeoning field of constitutive modelling of geomaterials, they are certainly important pointers to where the field is headed. With unlimited resources of computation and unfettered access to experimental data, modelling complex geomaterials and its various facets is becoming increasingly important. Given the myriad constraints that geotechnical engineers face in designing various infrastructure elements, a deep understanding of constitutive modelling of geomaterials and its implementation into boundary value problems is paramount. With physicists, rheologists, mining engineers, contributing to modelling of geomaterials at multiple length scales, interdisciplinarity is the way forward. 